\title{
Ketoacidosis Secondary to Dento-Alveolar Infection in a Diabetic Pediatric Patient
}

\author{
Sahar Kadri, Dorsaf Touil, Yosri Abdmouleh, Latifa Hammouda, Fatma Hannachi, \\ Lamia Oulha, and Nabiha Douki
}

\section{ABSTRACT}

Diabetic ketoacidosis (DKA) is a common complication of type I and occasionally type II diabetes mellitus. Patients with DKA require intensive multidisciplinary care involving dental surgeons and endocrine physicians in a hospital setting. The association between diabetes mellitus and oral infections is very common and infection is a well-recognized trigger of DKA. Yet very few cases of DKA secondary to dental infections have been reported in the literature. We, herein, report an interesting case of a pediatric patient presenting with ketoacidosis secondary to acute cervical adenophlegmon of dental origin.

Keywords: Decompensation, Dentistry, Diabetes type I, Infection, Ketoacidosis, Lymphadenitis.

\section{INTRODUCTION}

Diabetic ketoacidosis (DKA) is a common complication of type I and occasionally type II diabetes mellitus. In pediatric patients, this condition is defined when blood glucose level is over $11 \mathrm{mmol} / \mathrm{L}$, venous $\mathrm{pH}$ is below 7.3, and in the presence of either ketonemia or moderate to high ketonuria [1].

This condition, together with the major complication of cerebral edema, remains the most important cause of mortality and severe morbidity in children with diabetes, with a mortality rate ranging between 5 and $10 \%$ [2].
Published Online: December 08, 2021

ISSN: $2684-4443$

DOI: 10.24018 /ejdent.2021.2.6.120

\section{Sahar Kadri*}

Research Laboratory LR12ES11Dental Faculty of Monastir, University of Monastir, Tunisia.

(e-mail: kadrisahar1995@gmail.com)

Dorsaf Touil

Research Laboratory LR12ES11Dental

Faculty of Monastir, University of Monastir, Tunisia.

(e-mail: touil.oueslati.dorsaf@gmail.com) Yosri Abdmouleh

Research Laboratory LR12ES11Dental Faculty of Monastir, University of Monastir, Tunisia.

(e-mail: abdmoulehyosri94@gmail.com) Latifa Hammouda

Research Laboratory LR12ES11Dental Faculty of Monastir, University of Monastir, Tunisia.

(e-mail: hamoudalatifa1994@gmail.com) Fatma Hannachi

Research Laboratory LR12ES11Dental Faculty of Monastir, University of Monastir, Tunisia.

(e-mail: fhannachi94@gmail.com) Lamia Oualha

Research Laboratory LR12ES11Dental Faculty of Monastir, University of Monastir, Tunisia.

(e-mail: lamia.oualha@gmail.com)

Nabiha Douki

Research Laboratory LR12ES11Dental Faculty of Monastir, University of Monastir, Tunisia.

(e-mail: nabiha.douki@gmail.com)

*Corresponding Author

Patients with DKA require intensive multidisciplinary care involving dental surgeons and endocrine physicians in a hospital setting. The clinical signs of DKA include dehydration that may be difficult to detect, tachycardia, deep sighing respiration with typical ketotic smelling breath, nausea, vomiting, abdominal pain, drowsiness, progressive reduction in the level of consciousness, and eventually loss of consciousness [3].

The association between diabetes mellitus and oral infections is very common. However, the etiopathology of this association is still debatable. Joshi et al. [2] reported a 
lack of clinical evidence supporting that diabetic patients are more susceptible to infection than nondiabetic patients. Nevertheless, infection is a well-recognized trigger of DKA. Many studies have investigated the role of infection as a trigger of DKA and the impact of antimicrobial treatment in the management of this complication. Moreover, dentoalveolar abscess has previously been reported to be a precipitating cause of DKA in diabetes patients [4].

Yet very few cases of DKA secondary to dental infections have been reported in the literature.

Awareness of the potential consequences of oral infection in diabetic patients may allow dentists and dental specialists to promptly identify and therefore prevent and manage DKA at an early stage.

We, herein, report an interesting case of a pediatric patient presenting with ketoacidosis secondary to acute cervical adenophlegmon of dental origin.

\section{OBSERVATION}

A 10-year-old male patient receiving insulin treatment was referred to the department of oral medicine at the university hospital Sahloul for acute cervical adenophlegmon. He had been diagnosed with type I diabetes at the age of 5. A possible dental origin was searched.

Physical examination showed an ill-looking boy on a wheelchair. The patient was hospitalized due to severe systemic signs and symptoms marked with fever, dehydration, chills, lethargy, nausea, vomiting and abdominal pain, and drowsiness during the previous few days. An important weight loss was also noted. The patient was rehydrated with Intravenous normal saline together with insulin infusion.

\begin{tabular}{cc} 
TABLE I: BIOLOGICAL INVESTIGATIONS REVEALED: \\
\hline Hb1Ac (\%] & 11 \\
Glycaemia (g/l) & 4.9 \\
Ketonemia (mmol/l) & 5.2 \\
ketonuria & ++ \\
\hline
\end{tabular}

The patient presented with persistent spiking fever beginning 3 days previously, progressive left face and neck swelling, redness, and pain associated with a $4 \mathrm{~cm}$ length painful and indurated sub-mandibular lymphadenitis (Fig. 1).

Nuchal soreness, especially when turning the head, dysphagia, continuous purulent oozing from his decayed mandibular left molar (36), as well as severe trismus were noted (Fig. 2).

Oral examination revealed a typical ketotic halitosis. The tooth 36 was severely decayed. A fluctuant red swelling of $3 \mathrm{~cm}$ in diameter blanching to pressure was present in the buccal sulcus of the tooth 36. Diagnosis of left buccal sulcus abscess of the tooth 36 aggravated by acute submandibular suppurative lymphadenitis was retained. Cervical ultrasound showed a $10 \mathrm{~mm}$ hypertrophied jugulo-carotid adenomegaly with multiple reactive bilateral lymph nodes (Fig. 4).

Given the severity of the mouth opening restriction, no dental treatment was possible. Intravenous antibiotic therapy (Ampicillin and Metronidazole) and pain killer (Perfalgan) were administrated. Endodontic treatment was postponed for 48 h. Meanwhile, surgical drainage of the suppurative cervical lymphadenitis was performed.

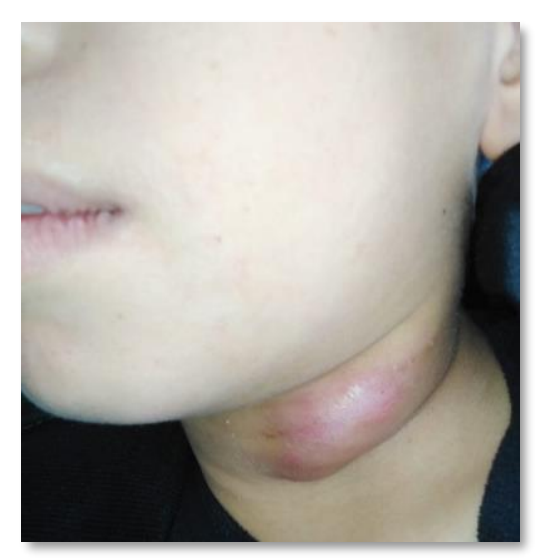

Fig. 1. A 4-cm length painful and indurated sub-mandibular lymphadenitis.

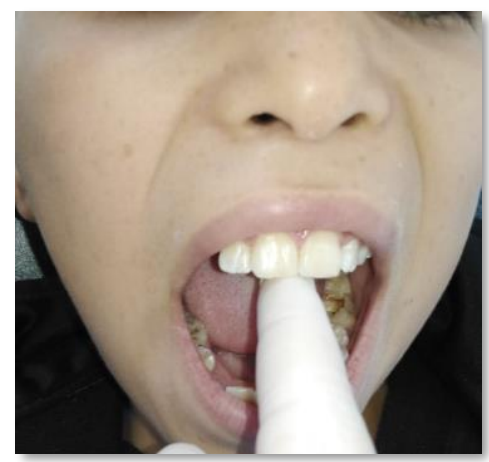

Fig. 2. Severe restriction of the mouth opening.

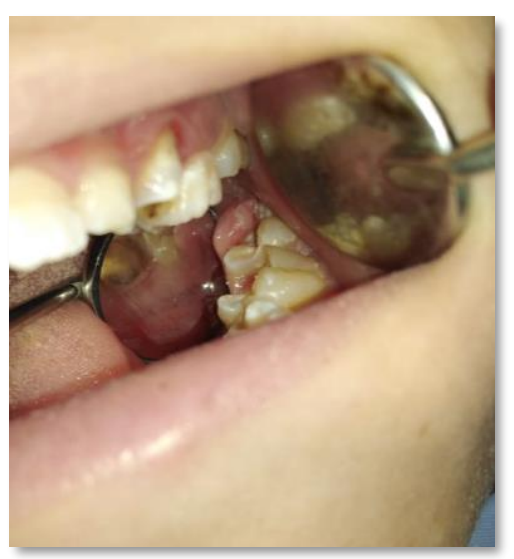

Fig. 3. A fluctuant, red swelling blanching to pressure was present in the buccal sulcus of the decayed tooth 36 .
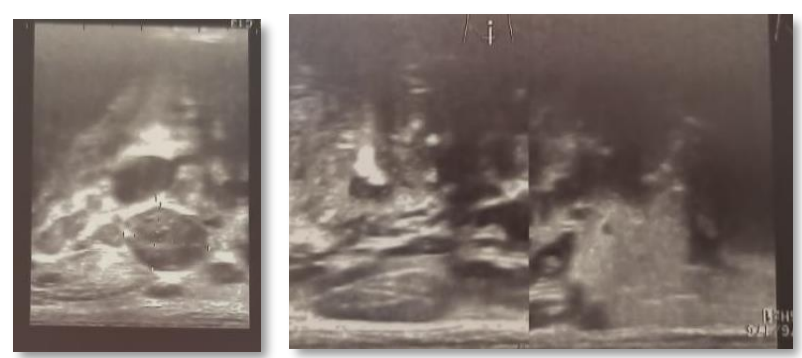

Fig. 4. Longitudinal sonogram showing multiple reactive lymph nodes (arrows). The lymph nodes are hypoechoic, oval-shaped, and they maintain their echogenic hilus (arrowheads).

Two days later, improvement of the mouth opening was noted (Fig. 5). Conservative treatment of the tooth 36 was preferred to extraction. A rubber dam was therefore set, the access cavity was opened, and all the necrotic tissue was gently removed using K-Files 8, 10, 15, 20 (Fig. 6). K-files were used only for root canal debridement. Important pus 
drainage was observed during all the procedure. Copious irrigation was later performed with $2.5 \%$ sodium hypochlorite. Dressing with Calcium hydroxide was used as an intra-canal medicament.

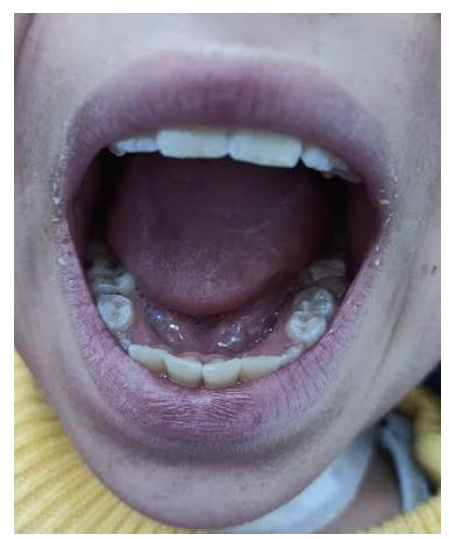

Fig. 5. The patient's follow up was marked with an improvement in his overall health status; restoration of a normal mouth opening.

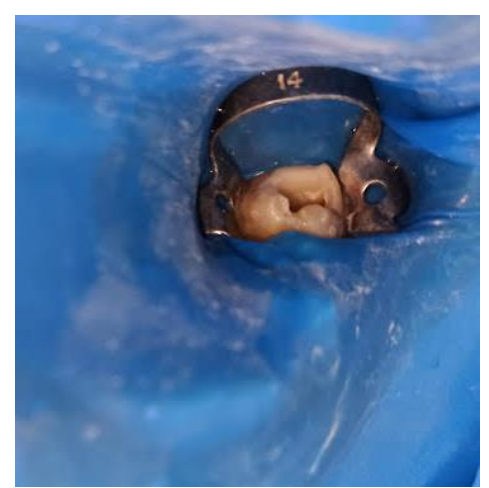

Fig. 6. Endodontic treatment of the causal tooth (36); drainage of pus through the pulp.

One week later, resolution of metabolic acidosis was observed. The patient presented with an improvement of his overall health status, especially pain, fever, and mouth opening. Clinical and radiographic follow-up visits were scheduled.

\section{DISCUSSION}

Diabetic ketoacidosis is considered as the most common and severe complication of diabetes in children and adolescents [1]. It is the result of severe or absolute insulin deficiency, which explains its increased incidence in patients with type I diabetes compared to those with type II diabetes. [6], [8].

The classic clinical signs of DKA include polyuria, polydipsia, polyphagia, and weight loss. However, Clinical signs may progress rapidly and include vomiting, abdominal pain, dehydration, weakness, and lethargy.

The main important factors responsible for triggering ketoacidosis include non-compliance with insulin therapy and infections [7]. However, glycemic control in the pediatric population with diabetes is hard to be obtained. A metaanalysis conducted by Korey et al. showed that adherence is linked to glycemic outcomes in pediatric type I diabetes. In this regard, adherence to an intensive insulin regimen leads to improvement in glycemic control and subsequently a reduced risk of the long-term complications of the disease. The authors concluded that these findings emphasize the benefit of adherence to children and adolescents with type I diabetes. [8] Nevertheless, infection, accounting for30-50\% of cases, remains the most common precipitating cause for DKA. Urinary tract infection and pneumonia account for the majority of infections, followed by intercurrent illnesses, such as myocardial ischemia and pancreatitis. The mechanism can be explained by the deficiency of circulating insulin and the increased levels of the counter regulatory hormones, such as Glucagon, catecholamines, cortisol, and growth hormone caused by stress and local infection.

Very few cases of DKA in children caused by dental infection have been reported in the literature. A. Chandu et al. reported two cases of DKA in adult patients with type I diabetes caused by dental infections. The authors concluded the need for training to dental professionals to be able to recognize the signs and symptoms of DKA in their patients. Indeed, quick recognition and early referral minimize potential complications and mortality [4].

In our case, the young patient presented with severe symptoms of DKA in addition to symptoms of dental infection related to the decayed tooth. The dento-alveolar abscess was aggravated by an acute lymphadenitis which marked the spread of the dental infection to the neck spaces. In this regard, diffused nuchal and facial infections in children have been the subject of multiple recent studies investigating the increased incidence of this complication in young patients compared to the elderly population. Pediatric odontogenic infections and their management continue to be a challenge for clinicians because they are uncommon. Hence, children with facial swelling, pain, and fever should be managed immediately. Failure to recognize and manage infection at an early stage can lead to serious complications, as in our case. Hence, accurate diagnosis and administration of the appropriate drugs as well as surgical therapies should result in rapid resolution of the symptoms. If the initial treatment response is poor, alternative infectious causes and therapies should be considered, along with hospitalization to manage the infection.

Diagnosis of the odontogenic origin of the infection is often made based on the clinical symptoms and the radiographic investigations, such as panoramic radiograph, CBCT, and ultrasound [9].

In fact, ultrasound is a useful imaging tool in the initial evaluation of cervical lymph nodes because it has a high sensitivity (98\%) and specificity (95\%). In our case, Ultrasound was indicated, and it revealed multiple reactive lymph nodes, confirming the reactive origin of lymphadenitis.

Successful treatment of DKA requires frequent patients' monitoring, correction of hypovolemia and hyperglycemia, replacement of electrolyte loss, and careful search for the precipitating cause. In our case, the dento-alveolar abscess was believed to be the precipitating cause. Hence, endodontic treatment of the decayed tooth (36) was performed rapidly under systemic antibiotics combined with surgical drainage of the cervical collection.

The patient's follow-up was marked with an improvement in his overall health status, especially pain and fever. Correction of his biological markers was also noted.

Follow-up sessions were scheduled. Monitoring dental 
infections in diabetic children must be established with the parents' collaboration in order to maintain good oral hygiene and prevent relapses and severe complications of decompensated diabetes.

\section{CONFLICT OF INTEREST}

All authors declare that they do not have any conflict of interest.

\section{REFERENCES}

[1] Raghupathy P. Diabetic ketoacidosis in children and adolescents. Indian $J$ Endocrinol Metab. 2015;19 (Suppl 1):S55-S57. doi:10.4103/2230-8210.155403.

[2] Joshi N, Caputo GM, Weitekamp MR, Karchmer AW. Infections in patients with diabetes mellitus. N Engl J Med. 1999 Dec 16;341(25):1906-12. doi: 10.1056/NEJM199912163412507. PMID: 10601511.

[3] Castellanos L, Tuffaha M, Koren D, Levitsky LL. Management of Diabetic Ketoacidosis in Children and Adolescents with Type 1 Diabetes Mellitus. Paediatr Drugs. 2020 Aug;22(4):357-367. doi: 10.1007/s40272-020-00397-0. PMID: 32449138.

[4] Chandu A, Macisaac RJ, Smith AC, Bach LA. Diabetic ketoacidosis secondary to dento-alveolar infection. Int J Oral Maxillofac Surg. 2002 Feb;31(1):57-9. doi: 10.1054/ijom.2001.0140. PMID: 11936401.

[5] Raghupathy P. Diabetic ketoacidosis in children and adolescents. Indian journal of endocrinology and metabolism 2015; 19(Suppl 1), S55-S57. https://doi.org/10.4103/2230-8210.155403.

[6] A. Chandu, R.J. MacIsaac, A.C.H. Smith, L.A. Bach, Diabetic ketoacidosis secondary to dento-alveolar infection. International Journal of Oral and Maxillofacial Surgery, 2002;31(1):57-59, ISSN 0901-5027.

https://doi.org/10.1054/ijom.2001.0140.(https://www.sciencedirect.co $\mathrm{m} /$ science/article/pii/S0901502701901407).

[7] Shahgoli, S., Shapiro, R., \& Best, J. A. A dentoalveolar abscess in a pediatric patient with ketoacidosis caused by occult diabetes mellitus: a case report. Oral surgery, oral medicine, oral pathology, oral radiology, and endodontics, 1999; 88(2):164-166. https://doi.org/10.1016/s1079-2104(99)70111-3.

[8] Korey K. Hood, Claire M. Peterson, Jennifer M. Rohan and Dennis Drotar. Association Between Adherence and Glycemic Control in Pediatric Type 1 Diabetes: A Meta-analysis. Pediatrics, 2009; 124; e1171-e1179; originally published online Nov 2, 2009; DOI: 10.1542/peds.2009-0207.

[9] Walk EL, McLaughlin S, Coad J, Weed SA. Use of High Frequency Ultrasound to Monitor Cervical Lymph Node Alterations in Mice. PLOS ONE, 2014;9(6):e100185.

https://doi.org/10.1371/journal.pone.0100185). 